Review Article

\title{
Impaired Autophagy of GABAergic Interneurons in Neuropathic Pain
}

\author{
Yuhua Yin $\mathbb{D}^{1,2}$ Min-Hee Yi ${ }^{1},^{3}$ and Dong Woon Kim $\mathbb{D}^{1,2}$ \\ ${ }^{1}$ Department of Medical Science, Chungnam National University School of Medicine, Daejeon 35015, Republic of Korea \\ ${ }^{2}$ Department of Anatomy and Cell Biology, Brain Research Institute, Chungnam National University School of Medicine, Daejeon \\ 35015, Republic of Korea \\ ${ }^{3}$ Department of Neurology, Mayo Clinic, Rochester, MN 55905, USA
}

Correspondence should be addressed to Dong Woon Kim; visnu528@cnu.ac.kr

Received 20 July 2018; Accepted 12 September 2018; Published 25 September 2018

Academic Editor: Parisa Gazerani

Copyright (C) 2018 Yuhua Yin et al. This is an open access article distributed under the Creative Commons Attribution License, which permits unrestricted use, distribution, and reproduction in any medium, provided the original work is properly cited.

\begin{abstract}
Neuropathic pain (NP) is caused by lesions of the peripheral fibers and central neurons in the somatosensory nervous system and affects $7-10 \%$ of the general population. Although the distinct cause of neuropathic pain has been investigated in primary afferent neurons over the years, pain modulation by central sensitization remains controversial. NP is believed to be driven by cell typespecific spinal synaptic plasticity in the dorsal horn. Upon intense afferent stimulation, spinothalamic tract neurons are potentiated, whereas GABAergic interneurons are inhibited leading to long-term depression. Growing evidences suggest that the inhibition of GABAergic neurons plays pivotal roles in the manifestation of neuropathic and inflammatory pain states. Downregulation of GABA transmission and impairment of GABAergic interneurons in the dorsal horn are critical consequences after spinal cord and peripheral nerve injuries. These impairments in GABAergic interneurons may be associated with dysfunctional autophagy, resulting in neuropathic pain. Here, we review an emerging number of investigations that suggest a pivotal role of impaired autophagy of GABAergic interneurons in NP. We discuss relevant research spurring the development of new targets and therapeutic agents of NP and emphasize the need for a multidisciplinary approach to manage NP in the future.
\end{abstract}

\section{Introduction}

More than 20 million individuals have neuropathic pain (NP) worldwide and suffer from highly acute diseases, greater healthcare costs, and lower quality of life [1-3]. In some cases, the most severe NP leads to increased episodes of depression and suicide [4-6]. The etiology of NP is broad and classified into central nervous system (CNS), peripheral nervous system, and complex neuropathic disorders, including complex regional pain syndrome types I (reflex sympathetic dystrophy) and II. Complex regional pain syndrome refers to a chronic pain condition characterized by progressively worsening spontaneous regional pain in the absence or presence of peripheral nerve damage. The traditional treatments for chronic pain, such as medications, massage therapy, acupuncture, electrical stimulation, nerve blocks, and surgery, cause severe side effects and fail to reduce or eliminate NP [7]. Further research is needed to understand the mechanisms underlying NP to allow for the design of individual and rational treatment strategies but also for the development of optimal therapeutic drugs.

Changes in the properties of neurons in the CNS can result in central sensitization. Even normal inputs that usually evoke innocuous sensations can lead to pain hypersensitivity due to the changes in the sensory response [8]. Moreover, there is evidence that central sensitization refers to subsequent peripheral nerve injury (PNI) or neuropathy [9]. In the past, many researchers believed that NP following PNI was due to the changes in the CNS and peripheral nervous system (PNS) neurons and their functions. Accordingly, many studies have focused on spinal dorsal neurons to elucidate the mechanism of NP. These studies have suggested that NP is a consequence of the aberrant response of the sensory neurons in the dorsal horn to the PNS inputs, anatomical reorganization of the pain pathway, 
as well as attenuated microglial activation and inhibitory processes [10]. A number of mechanisms have been suggested for central sensitization, among which there is strongest evidence for the dysfunction of GABAergic interneurons and their role in modifying synaptic transmission of pain signaling pathways in response to PNI [11-13].

Under normal circumstances, inhibitory interneurons continuously release GABA to decrease the excitability of lamina I output neurons and modulate pain transmission. However, this inhibition can be lost after an injury, resulting in hyperalgesia $[10,14]$. This may occur as a result of changes in loss of GABAergic function or apoptosis. Additionally, disinhibition may enable nonnociceptive myelinated $A \beta$ primary afferents to engage the pain transmission circuitry, leading to a perception of pain in patients in response to normally innocuous stimuli. It remains highly controversial to link the changes of cell types and cell death in the spinal dorsal horn with NP. It was reported that partial PNI leads to neuronal cell death in the dorsal horn [15]. Spinal cord injury-induced GABAergic interneuron cell death and their decreased number in the dorsal horn diminishes GABAergic tone and NP [14]. However, it has also been reported that the number of GABAergic interneurons and the synaptic boutons at $\mathrm{GABA}_{\mathrm{A}}$ synapses, as well as the expression levels of intracellular GAD65, GAD67, GABA, GABA transporters 1 and 3 , and $\mathrm{GABA}_{\mathrm{A}}$ and $\mathrm{GABA}_{\mathrm{B}}$ receptors can all remain unaltered [16-20].

The differences in these reports may be reconciled when considering time-dependent changes due to autophagy. Autophagy, a cellular self-digestion pathway involved in protein and organelle degradation, is known to be highly involved in various human diseases and physiology. Autophagy regulates cell homeostasis with a dual role in cell protection and cell death. However, some roles of autophagy are related to NP. We will discuss the importance of GABAergic neurons in NP and their regulation by autophagy in this review.

1.1. GABAergic Neuron Dysfunction in NP. Lamina II of the dorsal horn (substantia gelatinosa) is a major target of nociceptive primary afferents. Although excitatory and inhibitory interneurons show considerable heterogeneity, a morphological study reported that the islet cells are inhibitory, the radial and large vertical cells are excitatory, and some neurons with a morphological appearance of vertical cells are GABAergic [21]. It is estimated that approximately $30 \%$ of lamina II neurons are GABAergic, especially with enriched glycine. The rest appear to be glutamatergic and have high expression of vesicular glutamate transporter 2 [22-24].

GABAergic inhibition has been suggested to play a pivotal role in the manifestation of NP and inflammatory pain states [15], and GABAergic interneurons are densely innervated in lamina I giant project neurons [25]. As a result, $\mathrm{NP}$ occurs during pharmacological inhibition of spinal $\mathrm{GABA}_{\mathrm{A}}$ and $\mathrm{GABA}_{\mathrm{B}}$ receptor-mediated inhibitory transmission [26-28]. Furthermore, $\mathrm{GABA}_{\mathrm{A}}$ receptor-mediated postsynaptic currents were observed to be inhibited after substantially reducing the primary afferents in the substantia gelatinosa of the spinal dorsal horn [15]. Moreover, $\mathrm{GABA}_{\mathrm{B}}$ receptor-mediated inhibition was lost after spinal nerve injury, particularly in the central terminals of primary afferents, leading to allodynia or spontaneous pain behavior $[18,29]$.

Endoplasmic reticulum (ER) stress was shown to be related to cellular reactive oxygen species (ROS) production, followed by an impaired autophagic flux. This cascade of events contributes to central sensitization of GABAergic interneuron dysfunction [30-33]. In particular, ROS are involved in long-term depression of GABAergic interneurons in mice [11]. Scavenging ROS enhance GABA current, and applying hydrogen peroxide inhibits GABA current, indicating that GABA release is redox sensitive [12]. L4 and L5 bilateral dorsal rhizotomy increases extracellular concentrations of excitatory amino acids in the dorsal horn and reduces the sensory glutamate-induced decreases in the expression of GABA-synthesizing enzymes and presynaptic inhibition $[34,35]$. Taken together, both autophagic impairment and ER stress contribute to dysfunction of dorsal horn GABAergic neurons in patients with NP. Therefore, regulating GABAergic neurons and inhibiting their function in the superficial dorsal horn of the spinal cord [15] are attractive therapeutic strategies developed to treat NP.

1.2. Autophagic Impairment in NP. There are several mechanisms of programmed cell death, including apoptosis and autophagy. Apoptosis involves a cooperation among caspases, whereas autophagic cell death involves (but not necessarily by) autophagic vacuolization [36]. Most previous studies focused only on apoptosis, but the study by Berliocchi et al. introduced the role of disrupted autophagy in an NP model using spinal nerve ligation (SNL) [37]. LC3II and Beclin 1 protein levels were found to be elevated after SNL. We also first reported that LC3 and Beclin 1 levels were significantly increased in the L4-5 spinal cord segment ipsilateral to the injured side in rats after SNL compared with the sham group [20]. In addition, the levels of LC3 and Beclin 1 were also significantly increased in GABAergic interneurons of the spinal dorsal horn following PNI [20]. Taken together, these studies suggest that autophagy is involved in the induction and maintenance of NP. Furthermore, a disturbance in autophagy renders GABAergic interneurons vulnerable to PNI or NP stimuli.

1.3. Inhibition of Autophagic Induction by 3-Methyladenine (3-MA) Reduces NP Behavior. Autophagy is an evolutionarily conserved catabolic process that involves degradation of cytoplasmic components. Autophagy involves multiple proteins and signaling pathways that can be divided into four distinct steps: (1) induction, (2) assembly and formation of autophagosomes, (3) docking and fusion with lysosomal membranes, and (4) degradation [38]. Autophagic flux is defined as a measure of autophagic degradation activity [39]. Therapeutic strategies that inhibit autophagy-induced cell 
death have the potential to identify novel and effective targeted treatments for NP.

3-methyladenine (3-MA) inhibits the induction of autophagy by blocking the formation of autophagosomes and inhibiting class III phosphatidylinositol 3-kinases $[20,40]$. We injected 3-MA intrathecally to investigate whether inhibition of autophagic dysfunction would result in effective relief of NP. 3-MA treatment was found to significantly reduce mechanical allodynia in a timedependent manner during postoperative observations before decreasing to baseline on day 14 (Figure 1) [20]. These results suggest that autophagy is a central regulator in the pathogenesis of NP.

1.4. Inhibition of Autophagic Flux by Chloroquine Reduces NP Behavior. Berliocchi et al. suggested that autophagy is modulated differently depending on the experimental pain model by demonstrating LC3I, Beclin 1, and p62 levels modulated differently for chronic constriction injury (CCI) versus spared nerve injury [41]. As described above, p62/SQSTM1 is an autophagic substrate and a key LC3binding protein, which serves as a link between LC 3 and ubiquitinated proteins [42]. p62 and p62-bound polyubiquitinated proteins become incorporated into the completed autophagosome and are degraded in autolysosomes. Because of the correlation between modulation of autophagy and p62 levels, this substrate is considered a useful proxy for autophagic degradation [42-44], and p62 levels are often increased when autophagy is impaired [43]. We previously demonstrated the increased accumulation of p62 on the ipsilateral side of CCI-induced mice compared with their contralateral side, suggesting that CCI leads to a blockage of the final degradative steps of autophagy [45].

We also reported that a high tissue content of omega-3 polyunsaturated fatty acids (PUFAs) attenuates formalininduced pain sensitivity, microglial activation, inducible nitric oxide synthase expression, and phosphorylation of NR2B [46]. In addition, we also showed higher levels of LC3II and Beclin 1 but lower levels of p62 in fat-1 mice, suggesting that polyunsaturated fatty acid enrichment enhances the induction and flux of autophagy. In that experiment, we demonstrated that brain-derived neurotrophic factor (BDNF) is expressed at higher levels in fat-1 mice, suggesting that BDNF and its associated proteins Akt and CREB, may be therapeutic targets for NP, because BDNF might be a neuroprotective factor by potentially upregulating autophagic flux activity.

Finally, we investigated blocking autophagy using chloroquine. Chloroquine reverses autophagy by accumulating in the lysosomes and disturbing vacuolar $\mathrm{H}^{+}$ATPase. This leads to a lysosomal acidification and results in autophagy inhibition [47]. Intrathecal injection of chloroquine into wild-type mice induces a significant reduction in the threshold of mechanical sensitivity [45]. Intrathecal chloroquine injection into naïve mice induces spinal accumulation of LC3 and p62, in parallel with significant mechanical hypersensitivity, thus confirming inhibition of autophagosome clearance and suggesting the participation of autophagy in spinal mechanisms of pain processing. Taken together, the analysis of LC3, Beclin 1, and p62 indicates that impaired autophagy in CCI-induced NP may result from blocking the late phase, rather than the induction phase of autophagic flux.

\section{Mitophagy Impairment in NP}

ROS are a major source of mitochondrial dysfunction and can induce mutations in mitochondrial DNA that result in protein deficiencies, restricted self-repair ability, and increase vulnerability of cells to ROS attack $[48,49]$. Indeed, ROS induces oxidative stress, which damages mitochondrial proteins and lipids [50, 51]. In particular, mitochondrial oxidative damage is usually caused by aging and is the most important risk factor in most cases of mitochondrial dysfunction [52]. As a result, mitophagy counteracts against excessive intracellular ROS and blocks ROS sources, which are important in the therapeutic management of NP [53].

Mitophagy is the selective autophagic degradation of mitochondria that occurs when mitochondria become defective following a damage or stress $[54,55]$, such as hypoxia, nutrient starvation, energy depletion, and other pharmacological or viral stimuli [56]. Increased generation of mitochondrial ROS detected in L5 dorsal horn neurons of SNLinduced neuropathic rats in dorsal horn neurons [57]. PINK1 is a neuroprotective protein involved in the activation of mitophagy by selectively accumulating in depolarized mitochondria and promoting PARK2/Parkin translocation [58]. We examined the expression of PINK1 in the spinal cord and found the number of immunoreactive cells and PINK1 protein expression were both increased significantly on the ipsilateral and contralateral sides of the spinal dorsal horn in wild-type mice. However, no such significant differences were observed in toll-like receptor 4 (TLR4) knockout mice [45]. PINK1 is expressed in neurons in the spinal dorsal horn, but not in astrocytes or microglia [45]. Thus, we demonstrated that mitophagy may play a role in NP-related processes, but further studies are needed to clarify the function of mitophagy in NP, especially in GABAergic interneurons.

\section{Impaired ER Stress in NP}

ER stress is caused by disruption of the structure and function of the ER [59]. The unfolded protein response (UPR) can be induced by a variety of cellular stresses, such as glucose deprivation, depletion of $\mathrm{ER} \mathrm{Ca}^{2+}$ stores, exposure to free radicals, and accumulation of unfolded or misfolded proteins $[60,61]$. UPR is mediated by three ER stress receptors: PKR-like ER kinase, inositol-requiring enzyme 1, and activating transcription factor-6. Both ER stress and the resulting UPR are part of the cellular homeostasis program that balance the protein turnover and synthesis $[62,63]$. However, if this equilibrium is disrupted, ER stress can activate programmed cell death pathways. Accordingly, activated ER stress pathways have been observed 


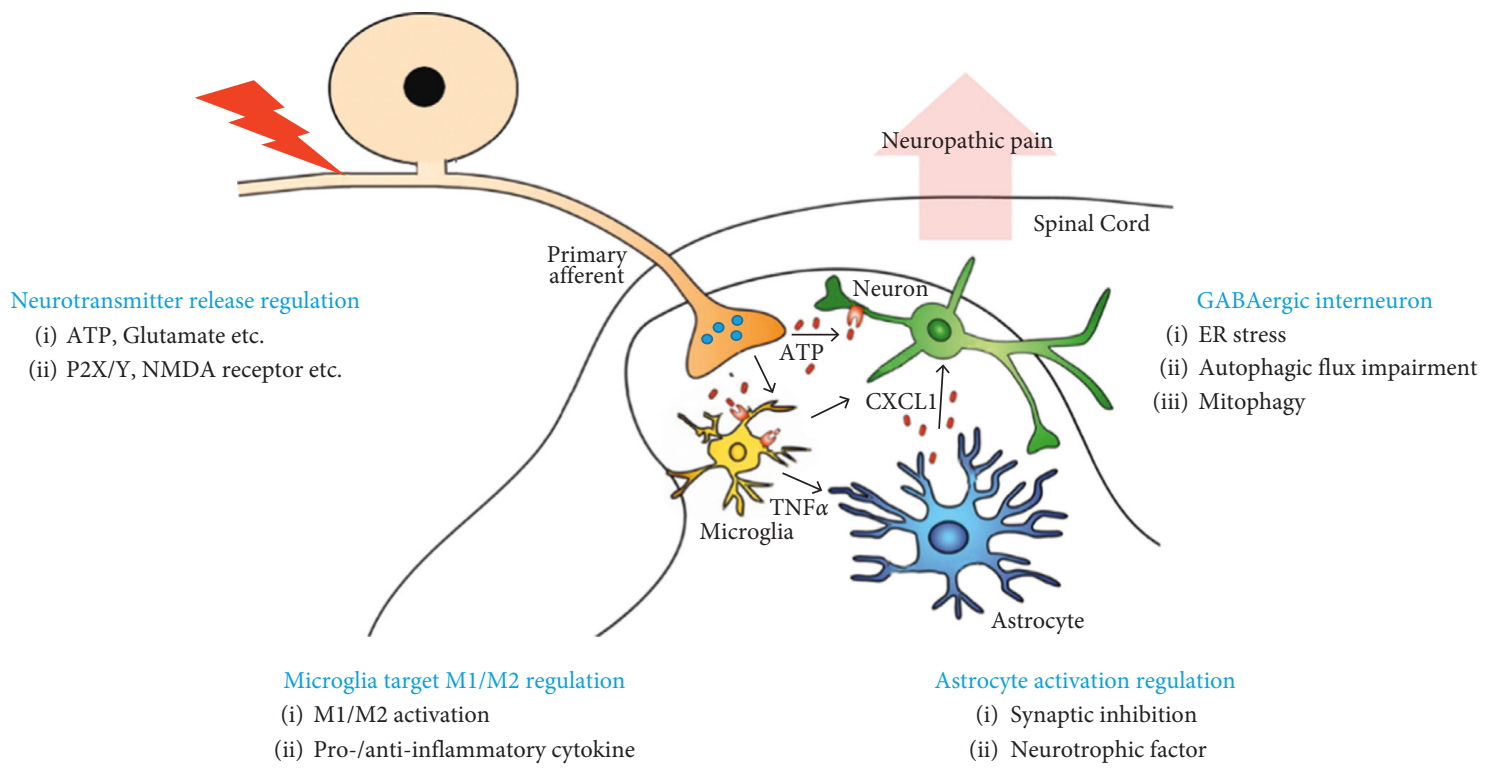

Figure 1: Therapeutic strategy to regulate GABAergic interneurons in spinal central sensitization. There is growing evidence that impaired autophagy and ER stress contribute to dysfunction of dorsal horn GABAergic neurons. Autophagosome accumulation and impairment of autophagic flux in GABAergic neurons make them susceptible to peripheral nerve injury or other pain stimuli. Therefore, there are several ways to control GABA neurons to control the spinal central sensitization that contributes to neuropathic pain. (1) Functional recovery of GABAergic interneurons, (2) neurotransmitter release regulation, (3) microglia targeted M1/2 polarization regulation, and (4) astrocyte targeted regulation.

in various disease pathogenesis, such as those of diabetes, cancers [64, 65], Alzheimer's disease, Parkinson's disease, amyotrophic lateral sclerosis, and prion diseases $[60,66]$. We also reported that the causes of ER stress in NP are related to autophagy and that autophagy was activated in an SNLinduced NP model [33].

As a common cytoprotective mechanism, autophagy controls homeostasis in various ways, such as protein and organelle turnover $[67,68]$. Moreover, autophagy is associated with the ER at various molecular levels, and ER stress induces autophagy in mammalian cells via several canonical UPR pathways $[69,70]$. We previously found that impaired ER stress in an SNL model and after ATF6 siRNA treatment significantly reduced NP-associated behaviors [33], indicating that ER stress is involved in the induction and maintenance of NP. Furthermore, we suggested that a disruption of the UPR pathway may render the neurons to become sensitive to PNI or NP stimuli. Further elucidation of the mechanisms of ER stress on GABAergic interneurons in the spinal cord during NP will be critical to guide the future development of novel pharmacological NP treatments.

\section{Nonneuronal Cell Regulation of NP}

In parallel with the progress achieved regarding these neuronal mechanisms, there has been an increased appreciation of the importance of nonneuronal cells, particularly glial cells such as microglia and astrocytes, to also play an important role in chronic pain. Over the past decade, there has been a dramatic increase in the number of pain research studies investigating the role of glia, which we briefly summarize in the following.
4.1. Activation of Spinal Microglia and NP. Microglia are resident macrophages of the spinal cord and brain. Although microglia comprise $5-12 \%$ of the cells in the CNS [71], abundant evidence supports their role in pathological pain [72-74]. Microglia are activated in the pathophysiological condition that occurs after PNI. [75] Once activated, microglia show morphological changes [76], increased expression of microglial markers, e.g., IBA1, MHC II, and CD $11 \mathrm{~b}$ [76-78], increased numbers (proliferation) [79-81], and increased phagocytosis [82]. TLR4 was previously reported to be primarily expressed in microglia; it was found that neuronal TLR4 regulates the proliferation of neural precursor cells during axonal growth, adult neurogenesis, and neuronal plasticity [83]. We reported previously that neuronal cells, and not astrocytes or microglia, of the CCIinduced NP spinal dorsal horn had impaired autophagy [45]. Our data showed that chloroquine treatment in TLR4 knockout mice attenuated the pain threshold in CCIinduced mechanical allodynia compared with TLR4 wildtype mice. Therefore, it is reasonable to conclude that the proinflammatory cytokines release in microglia, and the impaired autophagy in neurons synergistically contribute to pain sensory hypersensitivity via TLR4, suggesting TLR4mediated microglial activation may be indirectly coupled to autophagy.

4.2. Activation of Spinal Astrocytes. Increasing number of studies has shown that astrocytes are important in the development of chronic pain by regulating the extracellular concentrations of GABA released from neurons and glia by controlling its uptake [84-86]. Ischemia or hypoxia and gangliosides activate the autophagic/lysosomal pathway in 
astrocytes under oxidative stress [87]. Those authors reported that inhibiting this key system is protective. However, there have been no reports of activated autophagy in astrocytes of the spinal dorsal horn following NP or of autophagic impairment as an effective alternative treatment for NP. A tonic form of synaptic inhibition occurs in discrete regions of the CNS and has an important role in controlling neuronal excitability [88]. GABA in astrocytes is the major source of tonic inhibition in the cerebellum [89]. Although it is known that PNI alters astrocyte activity [90], it is unknown how astrocytes function as GABAergic and GABAceptive cells following PNI. Taken together, astrocytic GABA may actively participate in disinhibition of the neuronal networks in the spinal dorsal horn to regulate pain.

\section{Conclusions}

An accumulating body of literature has provided compelling evidence for the importance of neurons and glial cells in NP. The mechanisms regarding GABAergic interneuron dysfunction remain to be further elucidated. In this review, autophagic impairment of GABAergic interneurons in the spinal dorsal horn was identified as a new regulator of spinal neuronal circuits (Figure 1). We propose that impaired autophagy plays a critical role in spinal hypersensitivity, to which reduced GABAergic interneuronal activity is a key contributor in NP. This concept provides insight into the pathogenesis of NP and suggests potential strategies for developing new treatments for NP.

\section{Conflicts of Interest}

All the authors hereby declare that there are no potential conflicts of interest in the current study.

\section{Acknowledgments}

This research was supported by the Brain Research Program through the National Research Foundation of Korea (NRF) funded by the Ministry of Science, ICT and Future Planning (NRF-2016M3C7A1905074 and 2016R1A2B4009409). We are grateful to Prof. Yonghyun Kim (University of Alabama) for kind advice.

\section{References}

[1] B. H. McCarberg and R. Billington, "Consequences of neuropathic pain: quality-of-life issues and associated costs," American Journal of Managed Care, vol. 12, no. 9, pp. S263-S268, 2006.

[2] A. B. O'Connor, "Neuropathic pain: quality-of-life impact, costs and cost effectiveness of therapy," Pharmacoeconomics, vol. 27, no. 2, pp. 95-112, 2009.

[3] B. H. Smith, N. Torrance, M. I. Bennett, and A. J. Lee, "Health and quality of life associated with chronic pain of predominantly neuropathic origin in the community," Clinical Journal of Pain, vol. 23, no. 2, pp. 143-149, 2007.

[4] R. Alakhverdian and D. Koev, "Fibrinogen-fibrin degradation products in diabetes mellitus," Vŭtreshni Bolesti, vol. 26, no. 6, pp. 72-75, 1987.
[5] T. D. Hall, S. Shah, B. Ng et al., "Changes in mood, depression and suicidal ideation after commencing pregabalin for neuropathic pain," Australian Family Physician, vol. 43, no. 10, pp. 705-708, 2014.

[6] M. Risse and G. Weiler, "Histologic distribution pattern of thymus hemorrhages in sudden infant death," Beiträge zur gerichtlichen Medizin, vol. 46, pp. 351-355, 1988.

[7] T. Hashimoto, "Communication by personal computer," Josanpu Zasshi, vol. 42, no. 7, pp. 578-581, 1988.

[8] S. W. Snyder and M. S. Cardwell, "Neuromuscular blockade with magnesium sulfate and nifedipine," American Journal of Obstetrics and Gynecology, vol. 161, no. 1, pp. 35-36, 1989.

[9] D. Bridges, S. W. Thompson, and A. S. Rice, "Mechanisms of neuropathic pain," British Journal of Anaesthesia, vol. 87, no. 1, pp. 12-26, 2001.

[10] A. I. Basbaum, D. M. Bautista, G. Scherrer, and D. Julius, "Cellular and molecular mechanisms of pain," Cell, vol. 139, no. 2, pp. 267-284, 2009.

[11] A. Bittar, J. Jun, J. H. La, J. Wang, J. W. Leem, and J. M. Chung, "Reactive oxygen species affect spinal cell typespecific synaptic plasticity in a model of neuropathic pain," Pain, vol. 158, no. 11, pp. 2137-2146, 2017.

[12] D. W. Hogg, M. E. Pamenter, D. J. Dukoff, and L. T. Buck, "Decreases in mitochondrial reactive oxygen species initiate GABA(A) receptor-mediated electrical suppression in anoxiatolerant turtle neurons," Journal of Physiology, vol. 593, no. 10, pp. 2311-2326, 2015.

[13] Y. H. Kim, S. K. Back, A. J. Davies et al., "TRPV1 in GABAergic interneurons mediates neuropathic mechanical allodynia and disinhibition of the nociceptive circuitry in the spinal cord," Neuron, vol. 74, no. 4, pp. 640-647, 2012.

[14] J. G. Meisner, A. D. Marsh, and D. R. Marsh, "Loss of GABAergic interneurons in laminae I-III of the spinal cord dorsal horn contributes to reduced GABAergic tone and neuropathic pain after spinal cord injury," Journal of Neurotrauma, vol. 27, no. 4, pp. 729-737, 2010.

[15] K. A. Moore, T. Kohno, L. A. Karchewski, J. Scholz, H. Baba, and C. J. Woolf, "Partial peripheral nerve injury promotes a selective loss of GABAergic inhibition in the superficial dorsal horn of the spinal cord," Journal of Neuroscience, vol. 22, no. 15, pp. 6724-6731, 2002.

[16] M. J. Janssen, R. P. Yasuda, and S. Vicini, "GABA(A) receptor beta3 subunit expression regulates tonic current in developing striatopallidal medium spiny neurons," Frontiers in Cellular Neuroscience, vol. 5, p. 15, 2011.

[17] G. Miletic and V. Miletic, "Loose ligation of the sciatic nerve is associated with TrkB receptor-dependent decreases in KCC2 protein levels in the ipsilateral spinal dorsal horn," Pain, vol. 137, no. 3, pp. 532-539, 2008.

[18] E. Polgar and A. J. Todd, "Tactile allodynia can occur in the spared nerve injury model in the rat without selective loss of GABA or GABA(A) receptors from synapses in laminae I-II of the ipsilateral spinal dorsal horn," Neuroscience, vol. 156, no. 1, pp. 193-202, 2008.

[19] C. Sommer, A. Fahrner, and M. Kiessling, "Postischemic neuroprotection in the ischemia-tolerant state gerbil hippocampus is associated with increased ligand binding to inhibitory GABA(A) receptors," Acta Neuropathologica, vol. 105, no. 3, pp. 197-202, 2003.

[20] E. Zhang, M. H. Yi, Y. Ko et al., "Expression of LC3 and beclin 1 in the spinal dorsal horn following spinal nerve ligationinduced neuropathic pain," Brain Research, vol. 1519, pp. 31-39, 2013. 
[21] T. Yasaka, S. Y. Tiong, D. I. Hughes, J. S. Riddell, and A. J. Todd, "Populations of inhibitory and excitatory interneurons in lamina II of the adult rat spinal dorsal horn revealed by a combined electrophysiological and anatomical approach," Pain, vol. 151, no. 2, pp. 475-488, 2010.

[22] M. Mackie, D. I. Hughes, D. J. Maxwell, N. J. Tillakaratne, and A. J. Todd, "Distribution and colocalisation of glutamate decarboxylase isoforms in the rat spinal cord," Neuroscience, vol. 119, no. 2, pp. 461-472, 2003.

[23] D. J. Maxwell, M. D. Belle, O. Cheunsuang, A. Stewart, and R. Morris, "Morphology of inhibitory and excitatory interneurons in superficial laminae of the rat dorsal horn," Journal of Physiology, vol. 584, no. 2, pp. 521-533, 2007.

[24] A. J. Todd, D. I. Hughes, E. Polgar et al., "The expression of vesicular glutamate transporters VGLUT1 and VGLUT2 in neurochemically defined axonal populations in the rat spinal cord with emphasis on the dorsal horn," European Journal of Neuroscience, vol. 17, no. 1, pp. 13-27, 2003.

[25] E. Polgar, T. C. Sardella, M. Watanabe, and A. J. Todd, "Quantitative study of NPY-expressing GABAergic neurons and axons in rat spinal dorsal horn," Journal of Comparative Neurology, vol. 519, no. 6, pp. 1007-1023, 2011.

[26] S. P. Janssen, M. Truin, M. Van Kleef, and E. A. Joosten, "Differential GABAergic disinhibition during the development of painful peripheral neuropathy," Neuroscience, vol. 184, pp. 183-194, 2011.

[27] J. Lee, S. K. Back, E. J. Lim et al., "Are spinal GABAergic elements related to the manifestation of neuropathic pain in rat?," Korean Journal of Physiology and Pharmacology, vol. 14, no. 2, pp. 59-69, 2010.

[28] T. P. Malan, H. P. Mata, and F. Porreca, "Spinal GABA(A) and GABA(B) receptor pharmacology in a rat model of neuropathic pain," Anesthesiology, vol. 96, no. 5, pp. 1161-1167, 2002.

[29] M. P. Engle, M. Gassman, K. T. Sykes, B. Bettler, and D. L. Hammond, "Spinal nerve ligation does not alter the expression or function of GABA(B) receptors in spinal cord and dorsal root ganglia of the rat," Neuroscience, vol. 138, no. 4, pp. 1277-1287, 2006.

[30] S. S. Cao and R. J. Kaufman, "Endoplasmic reticulum stress and oxidative stress in cell fate decision and human disease," Antioxidants and Redox Signaling, vol. 21, no. 3, pp. 396-413, 2014.

[31] Y. Ge, Y. Jiao, P. Li et al., "Coregulation of endoplasmic reticulum stress and oxidative stress in neuropathic pain and disinhibition of the spinal nociceptive circuitry," Pain, vol. 159, no. 5, pp. 894-906, 2018.

[32] H. K. Kim, S. K. Park, J. L. Zhou et al., "Reactive oxygen species (ROS) play an important role in a rat model of neuropathic pain," Pain, vol. 111, no. 1-2, pp. 116-124, 2004.

[33] E. Zhang, M. H. Yi, N. Shin et al., "Endoplasmic reticulum stress impairment in the spinal dorsal horn of a neuropathic pain model," Scientific Reports, vol. 5, no. 1, article 11555, 2015.

[34] M. Mende, E. V. Fletcher, J. L. Belluardo et al., "Sensoryderived glutamate regulates presynaptic inhibitory terminals in mouse spinal cord," Neuron, vol. 90, no. 6, pp. 1189-1202, 2016.

[35] B. A. Rooney, E. D. Crown, C. E. Hulsebosch, and D. J. McAdoo, "Preemptive analgesia with lidocaine prevents failed back surgery syndrome," Experimental Neurology, vol. 204, no. 2, pp. 589-596, 2007.

[36] L. Galluzzi, I. Vitale, S. A. Aaronson et al., "Molecular mechanisms of cell death: recommendations of the nomenclature committee on cell death 2018," Cell Death and Differentiation, vol. 25, no. 3, pp. 486-541, 2018.

[37] L. Berliocchi, R. Russo, M. Maiaru, A. Levato, G. Bagetta, and M. T. Corasaniti, "Autophagy impairment in a mouse model of neuropathic pain,” Molecular Pain, vol. 7, p. 83, 2011.

[38] C. W. Wang and D. J. Klionsky, "The molecular mechanism of autophagy," Molecular Medicine, vol. 9, no. 3-4, pp. 65-76, 2003.

[39] B. Loos, A. du Toit, and J. H. Hofmeyr, "Defining and measuring autophagosome flux-concept and reality," Autophagy, vol. 10, no. 11, pp. 2087-2096, 2014.

[40] Y. T. Wu, H. L. Tan, G. Shui et al., "Dual role of 3-methyladenine in modulation of autophagy via different temporal patterns of inhibition on class I and III phosphoinositide 3kinase," Journal of Biological Chemistry, vol. 285, no. 14, pp. 10850-10861, 2010.

[41] L. Berliocchi, M. Maiaru, G. P. Varano et al., "Spinal autophagy is differently modulated in distinct mouse models of neuropathic pain," Molecular Pain, vol. 11, p. 3, 2015.

[42] G. Bjorkoy, T. Lamark, A. Brech et al., "p62/SQSTM1 forms protein aggregates degraded by autophagy and has a protective effect on huntingtin-induced cell death," Journal of Cell Biology, vol. 171, no. 4, pp. 603-614, 2005.

[43] M. Komatsu, S. Waguri, M. Koike et al., "Homeostatic levels of p62 control cytoplasmic inclusion body formation in autophagy-deficient mice," Cell, vol. 131, no. 6, pp. 1149-1163, 2007.

[44] S. Pankiv, T. H. Clausen, T. Lamark et al., "p62/SQSTM1 binds directly to Atg8/LC3 to facilitate degradation of ubiquitinated protein aggregates by autophagy," Journal of Biological Chemistry, vol. 282, no. 33, pp. 24131-24145, 2007.

[45] Y. Piao, D. H. Gwon, D. W. Kang et al., "TLR4-mediated autophagic impairment contributes to neuropathic pain in chronic constriction injury mice," Molecular Brain, vol. 11, no. 1, p. 11, 2018.

[46] E. Zhang, J. J. Kim, N. Shin et al., "High omega-3 polyunsaturated fatty acids in fat- 1 mice reduce inflammatory pain," Journal of Medicinal Food, vol. 20, no. 6, pp. 535-541, 2017.

[47] A. Nalbandian, K. J. Llewellyn, C. Nguyen, P. G. Yazdi, and V. E. Kimonis, "Rapamycin and chloroquine: the in vitro and in vivo effects of autophagy-modifying drugs show promising results in valosin containing protein multisystem proteinopathy," Plos One, vol. 10, no. 4, Article ID e0122888, 2015.

[48] I. Shokolenko, N. Venediktova, A. Bochkareva, G. L. Wilson, and M. F. Alexeyev, "Oxidative stress induces degradation of mitochondrial DNA," Nucleic Acids Research, vol. 37, no. 8, pp. 2539-2548, 2009.

[49] J. F. Turrens, "Mitochondrial formation of reactive oxygen species," Journal of Physiology, vol. 552, no. 2, pp. 335-344, 2003.

[50] S. Koirala, Q. Guo, R. Kalia et al., "Interchangeable adaptors regulate mitochondrial dynamin assembly for membrane scission," Proceedings of the National Academy of Sciences, vol. 110, no. 15, pp. E1342-E1351, 2013.

[51] B. Uttara, A. V. Singh, P. Zamboni, and R. T. Mahajan, "Oxidative stress and neurodegenerative diseases: a review of upstream and downstream antioxidant therapeutic options," Current Neuropharmacology, vol. 7, no. 1, pp. 65-74, 2009.

[52] B. A. Payne and P. F. Chinnery, "Mitochondrial dysfunction in aging: much progress but many unresolved questions," Biochimica et Biophysica Acta, vol. 1847, no. 11, pp. 13471353, 2015. 
[53] M. A. Arruda, P. Barcellos-de-Souza, A. L. Sampaio, A. G. Rossi, A. V. Graca-Souza, and C. Barja-Fidalgo, "NADPH oxidase-derived ROS: key modulators of hemeinduced mitochondrial stability in human neutrophils," Experimental Cell Research, vol. 312, no. 19, pp. 3939-3948, 2006.

[54] I. Kim, S. Rodriguez-Enriquez, and J. J. Lemasters, "Selective degradation of mitochondria by mitophagy," Archives of Biochemistry and Biophysics, vol. 462, no. 2, pp. 245-253, 2007.

[55] J. Lee, S. Giordano, and J. Zhang, "Autophagy, mitochondria and oxidative stress: cross-talk and redox signalling," Biochemical Journal, vol. 441, no. 2, pp. 523-540, 2012.

[56] S. Mai, B. Muster, J. Bereiter-Hahn, and M. Jendrach, "Autophagy proteins LC3B, ATG5 and ATG12 participate in quality control after mitochondrial damage and influence lifespan," Autophagy, vol. 8, no. 1, pp. 47-62, 2012.

[57] E. S. Park, X. Gao, J. M. Chung, and K. Chung, "Levels of mitochondrial reactive oxygen species increase in rat neuropathic spinal dorsal horn neurons," Neuroscience Letters, vol. 391, no. 3, pp. 108-111, 2006.

[58] V. Gelmetti, P. De Rosa, L. Torosantucci et al., "PINK1 and BECN1 relocalize at mitochondria-associated membranes during mitophagy and promote ER-mitochondria tethering and autophagosome formation," Autophagy, vol. 13, no. 4, pp. 654-669, 2017.

[59] D. Lindholm, H. Wootz, and L. Korhonen, "ER stress and neurodegenerative diseases," Cell Death and Differentiation, vol. 13, no. 3, pp. 385-392, 2006.

[60] K. M. Doyle, D. Kennedy, A. M. Gorman, S. Gupta, S. J. Healy, and A. Samali, "Unfolded proteins and endoplasmic reticulum stress in neurodegenerative disorders," Journal of Cellular and Molecular Medicine, vol. 15, no. 10, pp. 20252039, 2011.

[61] J. D. Malhotra and R. J. Kaufman, "The endoplasmic reticulum and the unfolded protein response," Seminars in Cell and Developmental Biology, vol. 18, no. 6, pp. 716-731, 2007.

[62] G. S. Hotamisligil, "Endoplasmic reticulum stress and the inflammatory basis of metabolic disease," Cell, vol. 140, no. 6 , pp. 900-917, 2010.

[63] Y. Kozutsumi, M. Segal, K. Normington, M. J. Gething, and J. Sambrook, "The presence of malfolded proteins in the endoplasmic reticulum signals the induction of glucoseregulated proteins," Nature, vol. 332, no. 6163, pp. 462464, 1988.

[64] J. H. Lin, P. Walter, and T. S. Yen, "Endoplasmic reticulum stress in disease pathogenesis," Annual Review of Pathology: Mechanisms of Disease, vol. 3, no. 1, pp. 399-425, 2008.

[65] S. Wang and R. J. Kaufman, "The impact of the unfolded protein response on human disease," Journal of Cell Biology, vol. 197, no. 7, pp. 857-867, 2012.

[66] R. A. Stetler, Y. Gan, W. Zhang et al., "Heat shock proteins: cellular and molecular mechanisms in the central nervous system," Progress in Neurobiology, vol. 92, no. 2, pp. 184-211, 2010.

[67] B. Levine and G. Kroemer, "Autophagy in the pathogenesis of disease," Cell, vol. 132, no. 1, pp. 27-42, 2008.

[68] T. Yorimitsu and D. J. Klionsky, "Autophagy: molecular machinery for self-eating," Cell Death and Differentiation, vol. 12, no. 2, pp. 1542-1552, 2005.

[69] H. O. Rashid, R. K. Yadav, H. R. Kim, and H. J. Chae, "ER stress: autophagy induction, inhibition and selection," Autophagy, vol. 11, no. 11, pp. 1956-1977, 2015.
[70] R. Sano and J. C. Reed, "ER stress-induced cell death mechanisms," Biochimica et Biophysica Acta (BBA)-Molecular Cell Research, vol. 1833, no. 12, pp. 3460-3470, 2013.

[71] L. J. Lawson, V. H. Perry, P. Dri, and S. Gordon, "Heterogeneity in the distribution and morphology of microglia in the normal adult mouse brain," Neuroscience, vol. 39, no. 1, pp. 151-170, 1990.

[72] P. M. Grace, M. R. Hutchinson, S. F. Maier, and L. R. Watkins, "Pathological pain and the neuroimmune interface," Nature Reviews Immunology, vol. 14, no. 4, pp. 217-231, 2014.

[73] R. R. Ji, T. Berta, and M. Nedergaard, "Glia and pain: is chronic pain a gliopathy?," Pain, vol. 154, no. 1, pp. S10-S28, 2013.

[74] R. R. Ji, A. Chamessian, and Y. Q. Zhang, "Pain regulation by non-neuronal cells and inflammation," Science, vol. 354, no. 6312 , pp. $572-577,2016$.

[75] M. Tsuda, K. Inoue, and M. W. Salter, "Neuropathic pain and spinal microglia: a big problem from molecules in "small" glia," Trends in Neurosciences, vol. 28, no. 2, pp. 101-107, 2005.

[76] N. P. Eriksson, J. K. Persson, M. Svensson, J. Arvidsson, C. Molander, and H. Aldskogius, "A quantitative analysis of the microglial cell reaction in central primary sensory projection territories following peripheral nerve injury in the adult rat," Experimental Brain Research, vol. 96, no. 1, pp. 19-27, 1993.

[77] D. E. Coyle, "Partial peripheral nerve injury leads to activation of astroglia and microglia which parallels the development of allodynic behavior," Glia, vol. 23, no. 1, pp. 75-83, 1998.

[78] L. Liu, E. Tornqvist, P. Mattsson et al., "Complement and clusterin in the spinal cord dorsal horn and gracile nucleus following sciatic nerve injury in the adult rat," Neuroscience, vol. 68, no. 1, pp. 167-179, 1995.

[79] R. W. Colburn, J. A. DeLeo, A. J. Rickman, M. P. Yeager, P. Kwon, and W. F. Hickey, "Dissociation of microglial activation and neuropathic pain behaviors following peripheral nerve injury in the rat," Journal of Neuroimmunology, vol. 79, no. 2, pp. 163-175, 1997.

[80] N. Gu, J. Peng, M. Murugan et al., "Spinal microgliosis due to resident microglial proliferation is required for pain hypersensitivity after peripheral nerve injury," Cell Reports, vol. 16, no. 3, pp. 605-614, 2016.

[81] Y. Yao, S. Echeverry, X. Q. Shi et al., "Dynamics of spinal microglia repopulation following an acute depletion," Scientific Reports, vol. 6, no. 1, article 22839, 2016.

[82] J. Gehrmann and R. B. Banati, "Microglial turnover in the injured CNS: activated microglia undergo delayed DNA fragmentation following peripheral nerve injury," Journal of Neuropathology and Experimental Neurology, vol. 54, no. 5, pp. 680-688, 1995.

[83] E. Okun, K. J. Griffioen, and M. P. Mattson, "Toll-like receptor signaling in neural plasticity and disease," Trends in Neurosciences, vol. 34, no. 5, pp. 269-281, 2011.

[84] J. Y. Chatton, L. Pellerin, and P. J. Magistretti, "GABA uptake into astrocytes is not associated with significant metabolic cost: implications for brain imaging of inhibitory transmission," Proceedings of the National Academy of Sciences, vol. 100, no. 21, pp. 12456-12461, 2003.

[85] A. Schousboe, A. Sarup, O. M. Larsson, and H. S. White, "GABA transporters as drug targets for modulation of GABAergic activity," Biochemical Pharmacology, vol. 68, no. 8, pp. 1557-1563, 2004.

[86] B. E. Yoon, J. Woo, and C. J. Lee, "Astrocytes as GABA-ergic and GABA-ceptive cells," Neurochemical Research, vol. 37, no. 11, pp. 2474-2479, 2012. 
[87] S. J. Lee, K. S. Cho, and J. Y. Koh, "Oxidative injury triggers autophagy in astrocytes: the role of endogenous zinc," Glia, vol. 57, no. 12, pp. 1351-1361, 2009.

[88] M. Farrant and Z. Nusser, "Variations on an inhibitory theme: phasic and tonic activation of GABA(A) receptors," Nature Reviews Neuroscience, vol. 6, no. 3, pp. 215-229, 2005.

[89] S. Lee, B. E. Yoon, K. Berglund et al., "Channel-mediated tonic GABA release from glia," Science, vol. 330, no. 6005, pp. 790-796, 2010.

[90] W. Wang, W. Wang, X. Mei et al., "Crosstalk between spinal astrocytes and neurons in nerve injury-induced neuropathic pain," PLoS One, vol. 4, no. 9, Article ID e6973, 2009. 


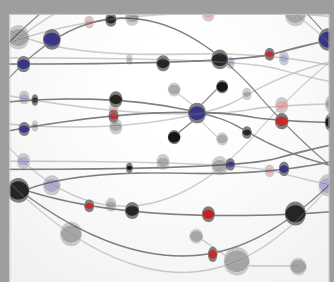

The Scientific World Journal
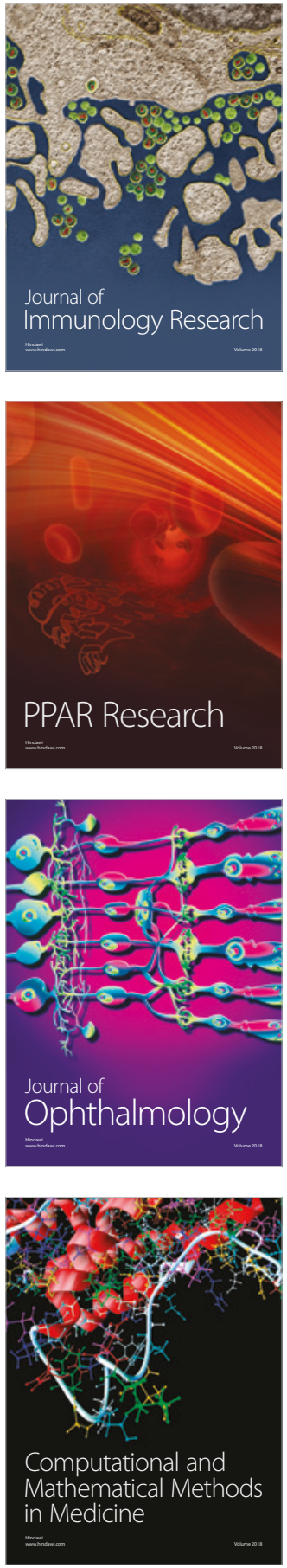

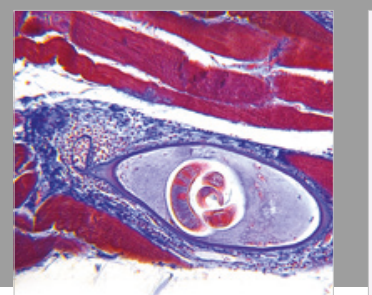

Gastroenterology Research and Practice

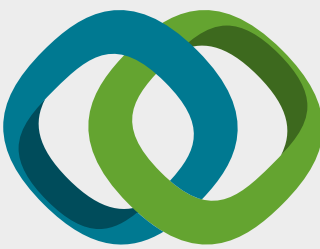

\section{Hindawi}

Submit your manuscripts at

www.hindawi.com
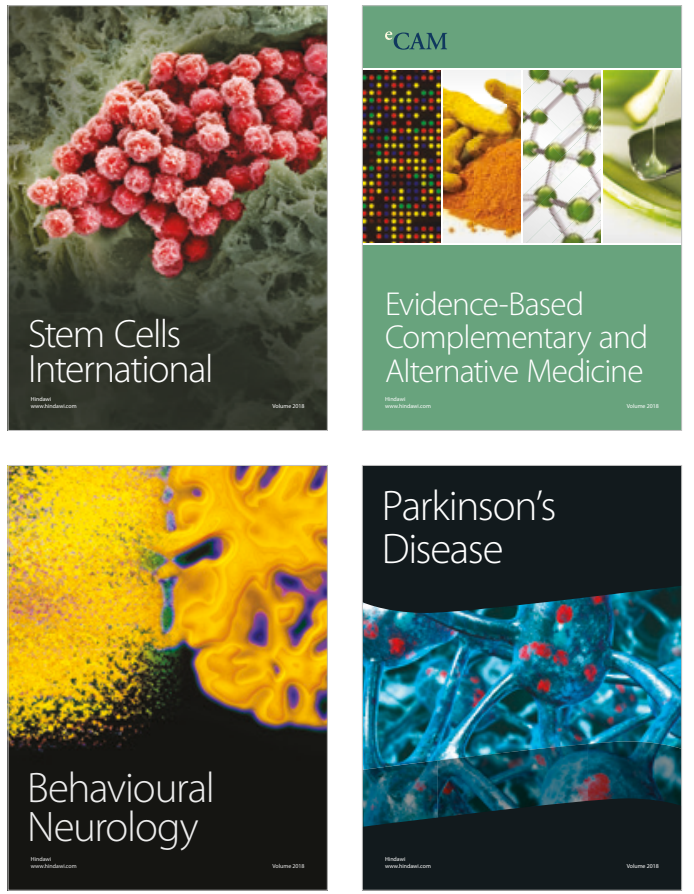

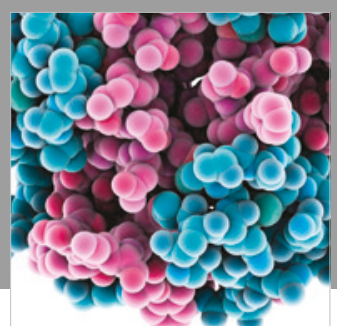

ournal of

Diabetes Research

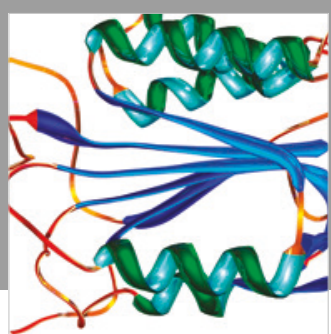

Disease Markers
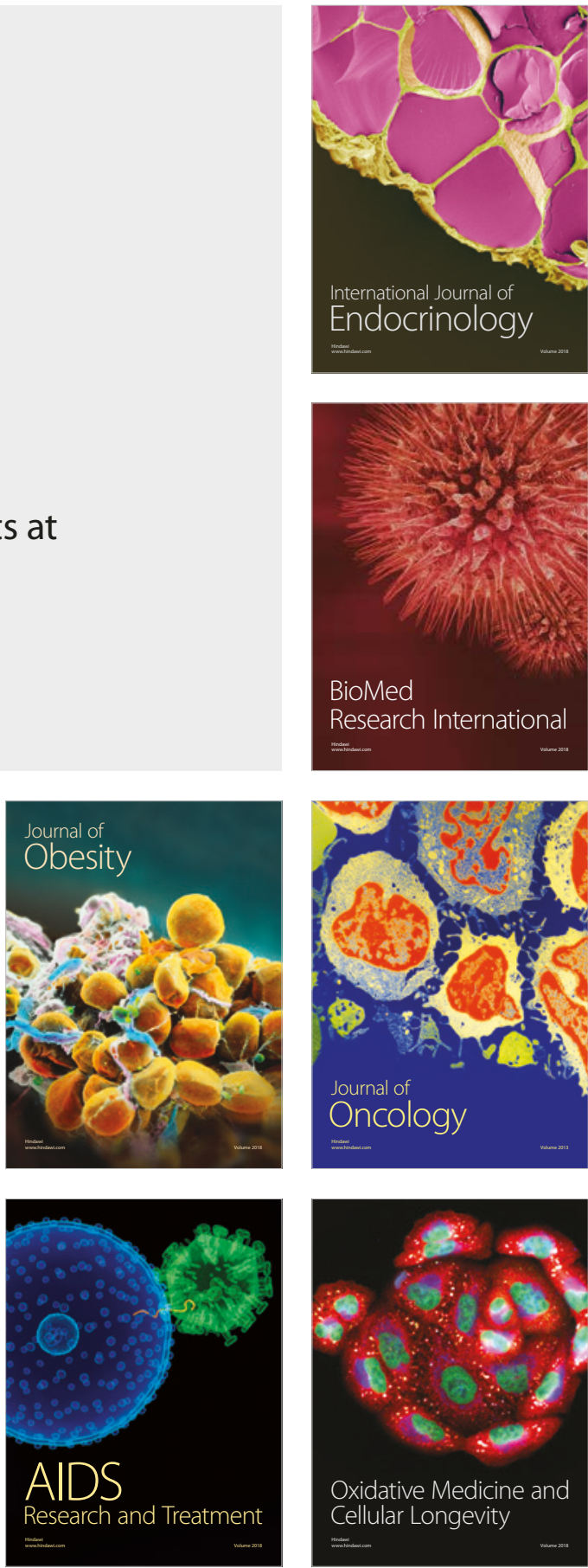